\title{
Synteny conservation between two distantly-related Rosaceae genomes: Prunus (the stone fruits) and Fragaria (the strawberry) Santiago Vilanova ${ }^{1,3}$, Daniel J Sargent ${ }^{2}$, Pere Arús ${ }^{1}$ and Amparo Monfort*1
}

Address: ${ }^{1}$ IRTA. Centre de Recerca en Agrigenòmica CSIC-IRTA-UAB, 08348 Cabrils, Spain, ${ }^{2 E a s t ~ M a l l i n g ~ R e s e a r c h ~(E M R), ~ E a s t ~ M a l l i n g, ~ K e n t, ~}$ ME19 6BJ, UK and ${ }^{3}$ Universidad Politécnica de Valencia, Centro de Conservación y Mejora de la Agrodiversidad Valenciana (COMAV), Spain

Email: Santiago Vilanova - sanvina@upvnet.upv.es; Daniel J Sargent - dan.sargent@emr.ac.uk; Pere Arús - pere.arus@irta.es; Amparo Monfort* - amparo.monfort@irta.es

* Corresponding author

Published: 18 June 2008

BMC Plant Biology 2008, 8:67 doi:10.1 | 86/147|-2229-8-67
Received: 31 October 2007

Accepted: 18 June 2008

This article is available from: http://www.biomedcentral.com/I47/-2229/8/67

(c) 2008 Vilanova et al; licensee BioMed Central Ltd.

This is an Open Access article distributed under the terms of the Creative Commons Attribution License (http://creativecommons.org/licenses/by/2.0), which permits unrestricted use, distribution, and reproduction in any medium, provided the original work is properly cited.

\begin{abstract}
Background: The Rosaceae encompass a large number of economically-important diploid and polyploid fruit and ornamental species in many different genera. The basic chromosome numbers of these genera are $x=7,8$ and 9 and all have compact and relatively similar genome sizes. Comparative mapping between distantly-related genera has been performed to a limited extent in the Rosaceae including a comparison between Malus (subfamily Maloideae) and Prunus (subfamily Prunoideae); however no data has been published to date comparing Malus or Prunus to a member of the subfamily Rosoideae. In this paper we compare the genome of Fragaria, a member of the Rosoideae, to Prunus, a member of the Prunoideae.
\end{abstract}

Results: The diploid genomes of Prunus $(2 n=2 x=16)$ and Fragaria $(2 n=2 x=14)$ were compared through the mapping of $7 \mathrm{I}$ anchor markers -40 restriction fragment length polymorphisms (RFLPs), 29 indels or single nucleotide polymorphisms (SNPs) derived from expressed sequence tags (ESTs) and two simple-sequence repeats (SSRs) - on the reference maps of both genera. These markers provided good coverage of the Prunus (78\%) and Fragaria (78\%) genomes, with maximum gaps and average densities of $22 \mathrm{cM}$ and $7.3 \mathrm{cM} /$ marker in Prunus and $32 \mathrm{cM}$ and $8.0 \mathrm{cM} / \mathrm{marker}$ in Fragaria.

Conclusion: Our results indicate a clear pattern of synteny, with most markers of each chromosome of one of these species mapping to one or two chromosomes of the other. A large number of rearrangements (36), most of which produced by inversions (27) and the rest (9) by translocations or fission/fusion events could also be inferred. We have provided the first framework for the comparison of the position of genes or DNA sequences of these two economically valuable and yet distantly-related genera of the Rosaceae.

\section{Background}

Genome comparisons based on the map position of homologous markers between different plant taxa have established that the genomes of species within families of the Plant Kingdom, such as Solanaceae [1,2], Poaceae [3],
Fabaceae [4] and Brassicaceae [5] differ in a limited number of chromosomal rearrangements, meaning that extensive chromosomal regions, and even entire chromosomes, are syntenic and colinear between different species [6]. However, conservation of synteny is greatly reduced 
when comparisons are made between members of different families, as shown through the comparison of the genomes of several crop species with that of Arabidopsis thaliana [7-9].

The Rosaceae encompass a large number $(3,000)$ of diploid and polyploid species [10] including important crops, such as those belonging to the genera Prunus (almond and all stone fruits: peach, apricot, cherry and plum), Malus (apple), Pyrus (pear), Rosa (rose), Rubus (raspberry) and Fragaria (strawberry). Their basic chromosome numbers are $x=7,8$ and 9 and all have a compact and relatively similar genome size that for diploid species ranges from $\sim 170 \mathrm{Mbp}$ in Fragaria $(2 \mathrm{n}=2 \mathrm{x}=14)$ [11] to $300 \mathrm{Mbp}$ in Prunus $(2 n=2 x=16)$ [12]. Most of the polyploid species within the family have a genome size approximately proportional to that of the diploid genomes from which they are composed, i.e. the amphidiploid apple $(2 n=2 x=34)$ has a genome size of $\sim 750 \mathrm{Mbp}$ [12] and the cultivated strawberry, an allooctoploid $(2 n=8 x=56)$, has a genome size of $\sim 800 \mathrm{Mbp}$ [11].

Comparative mapping has been performed to a limited extent in the Rosaceae [13]. The genus Prunus, from the subfamily Prunoideae, was the first to be studied, due to the existence of a high density reference map [14,15], based on a highly polymorphic interspecific (almond $x$ peach) $\mathrm{F}_{2}$ population and constructed with markers transferable to other species within the genus (mainly RFLPs and SSRs). The analysis of 16 published maps of Prunus species, each with at least 28 markers common to the reference map, established that the Prunus genome is essentially colinear and shared by all diploid species studied so far (peach, almond, apricot, cherry, myrobolan plum, $P$. davidiana, and P. ferganenesis) [13]. Comparisons between the genomes of apple and pear, which belong to the subfamily Maloideae, indicate a very high level of synteny, and no major chromosomal rearrangements can be deduced from studies of common markers mapped in these genera $[16,17]$. No data are currently published on synteny studies between members of the subfamily Rosoideae, which includes strawberry, rose and raspberry. There is however a partial comparison between the genomes of genera belonging to different sub-families: Prunus (Prunoideae) and Malus (Maloideae). This work was done with 34 markers (23 RFLPs and 11 isoenzymes) and, where comparisons were possible, a high level of synteny was detected, as well as at least one large-scale chromosomal rearrangement [14].

In this paper we have compared the genomes of Prunus and Fragaria. For this purpose, we have used the previously mentioned Prunus reference map and the Fragaria reference map [18], which is based on an interspecific dip- loid F. vesca $\times$ F. nubicola $\mathrm{F}_{2}$ population. The Fragaria map was constructed with mainly SSRs and markers derived from the growing information existing on expressed sequence tag (EST) sequences of different Rosaceae species, which are available through the Genome Database for the Rosaceae [19]. Here, we have established a framework with common markers covering both genomes and found that in spite of the conservation of large chromosomal fragments, as expected from confamilial species, many chromosomal rearrangements separate the diploid Fragaria and Prunus genomes, supporting their distant position within the family deduced from DNA sequence data [20].

\section{Results \\ Fragaria and Prunus maps}

In total, 71 anchor markers were available for the comparison between the diploid genomes of Prunus and Fragaria. These were: a) 40 RFLPs from the same number of probes found polymorphic out of 65 single-copy probes already mapped in Prunus (Table 1), b) 13 Fragaria ESTs obtained after selecting from 135 of them highly homologous to Prunus mapped ESTs out of the 515 ESTs of the Prunus transcript map (Table 2); these markers were selected by their position on the map as they fell in regions not covered with RFLPs or that were judged to be of interest for the genome comparison, c) eight Prunus ESTs selected from 13 ESTs of the transcript map that did not have sequence homology with known Fragaria ESTs (Table 2), d) eight Fragaria STSs obtained from genes of known function previously mapped in Fragaria [21] (Table 2), and e) two SSRs (Table 2).

Two mapping strategies were used in the placement of novel markers on both the $\mathrm{T} \times \mathrm{E}$ and $\mathrm{FV} \times \mathrm{FN}$ linkage maps (Table 3). For 55 markers: all 40 RFLPs, the eight Fragaria STSs, five of the Fragaria ESTs that displayed intron length polymorphisms and the two SSRs, were mapped by genotyping all individuals of the $\mathrm{FV} \times \mathrm{FN}$ population. The 16 remaining markers were mapped using the bin mapping approach where a set of six plants (the bin set) permit wselective' or 'bin' mapping using the diploid strawberry mapping population [22]. Only ten new markers were mapped in the T $\times$ E population: the eight Fragaria ESTs were bin mapped [15] and the two SSRs were mapped using the whole population.

The Fragaria map, constructed with MapMaker as described in the materials and methods, included 228 markers: 172 of the previous map [18], and 55 new markers studied here in the whole population. Three of the markers mapped in the map by Sargent et al. [18], CFVCT028, CFVCT05 and UFFxa03B05, could not be located using the conditions set for mapping in this work. The seven expected linkage groups (FG1-FG7) were 
Table I: RFLP probes mapped in Prunus and Fragaria Single-copy RFLP probes mapped in Prunus and used for mapping in Fragaria. RFLP position in the Prunus and Fragaria maps and estimated copy number in Fragaria

\begin{tabular}{|c|c|c|c|c|c|c|}
\hline \multirow[b]{2}{*}{ Marker } & \multirow[b]{2}{*}{ Accession No. } & \multicolumn{2}{|c|}{ Prunus } & \multicolumn{2}{|c|}{ Fragaria } & \multirow[b]{2}{*}{ Copy no. } \\
\hline & & $\mathrm{LG}^{\mathrm{a}}$ & $\mathrm{cM}$ & $\mathrm{LG}^{\mathrm{a}}$ & $\mathrm{cM}$ & \\
\hline AG53 & $\mathrm{BH} 023829$ & PGI & 2.5 & FG4 & 22.1 & 2 \\
\hline AC24 & $\mathrm{B} 1203138$ & PGI & 4.3 & FG4 & 20.3 & I \\
\hline AGI02 & $\mathrm{BH} 023845$ & PGI & 8.7 & FG6 & 63.4 & 2 \\
\hline PC78 & $\mathrm{B} 1203 \mid 48$ & PGI & 13.6 & FG4 & 31.1 & 1 \\
\hline AC32 & $\mathrm{B} 1203135$ & PGI & 25.8 & FG4 & 0 & 2 \\
\hline FG I 6 & $\mathrm{BH} 023876$ & PGI & 37 & $+c$ & - & - \\
\hline PC85 & n.d. ${ }^{b}$ & PGI & 37 & + & - & - \\
\hline PCI5 & $\mathrm{BI} 203140$ & PGI & 43 & + & - & - \\
\hline AG47 & $\mathrm{BH} 02386 \mathrm{I}$ & PGI & 43.7 & FG2 & 17.4 & 2 \\
\hline PC7 & n.d. & PGI & 44 & $++d$ & - & - \\
\hline FG36 & $\mathrm{BH} 023883$ & PGI & 65.1 & $\mathrm{FG} 2$ & 36.8 & I \\
\hline$A C I 3$ & $\mathrm{~B} 1203106$ & PG2 & 6 & + & - & - \\
\hline AC3I & BI203096 & PG2 & 7.9 & FG7 & 0 & 1 \\
\hline$A C 10$ & BI203087 & PG2 & 8.1 & FG4 & 13.5 & 2 \\
\hline PC5 & BI203107 & PG2 & 21 & + & - & - \\
\hline AG35 & $\mathrm{BH} 023896$ & PG2 & 25 & FG7 & 9 & 1 \\
\hline $\mathrm{ACl}$ & B1203097 & PG2 & 37 & ++ & - & - \\
\hline MC045 & BI203II7 & PG2 & 38 & FG7 & 81 & 2 \\
\hline Olel & $\times 78118$ & PG2 & 39 & + & - & - \\
\hline Omtl & $\times 83217$ & PG2 & 47.6 & FG7 & 89.6 & 1 \\
\hline $\mathrm{CCI} 25$ & n.d. & PG2 & 49 & + & - & - \\
\hline MCII5 & n.d. & PG3 & 0 & ++ & - & - \\
\hline FGI3 & n.d. & PG3 & 6 & ++ & - & - \\
\hline AG56 & n.d. & PG3 & 6.4 & FG6 & 65.2 & 1 \\
\hline AG7 & $\mathrm{BH} 023839$ & PG3 & 12 & + & - & - \\
\hline CCII6 & n.d. & PG3 & 22 & FG6 & 4.7 & I \\
\hline $\mathrm{CC2}$ & BI203070 & PG3 & 27.7 & FG6 & 43.7 & 2 \\
\hline $\mathrm{CC8}$ & Bl20309| & PG3 & 34.4 & FG6 & 81.9 & I \\
\hline AG 106 & $\mathrm{BH} 023814$ & PG3 & 37.1 & FG6 & 96 & 2 \\
\hline CC47 & $\mathrm{B} 1203119$ & PG4 & 5.4 & FG3 & 7.3 & I \\
\hline AG6 & BH023828 & PG4 & 24.1 & FG3 & 42.2 & 1 \\
\hline FG3 & $\mathrm{BH} 023837$ & PG4 & 37.7 & FG3 & 36.5 & 1 \\
\hline $\mathrm{PCI}$ & $\mathrm{B} 1203133$ & PG4 & 49.9 & FG3 & 48.2 & 1 \\
\hline PLG35 & n.d. & PG5 & 0 & $\mathrm{~m}^{\mathrm{e}}$ & - & - \\
\hline AC49 & BI203072 & PG5 & 15.2 & FG5 & 0 & I \\
\hline $\mathrm{PCI} 4$ & $\mathrm{~B} 1203132$ & PG5 & 20.6 & FG5 & 19.1 & I \\
\hline AG33 & $\mathrm{BH} 02382 \mathrm{I}$ & PG5 & 49.1 & FG5 & 56.4 & I \\
\hline AG 13 & n.d. & PG6 & 3 & + & - & - \\
\hline AG54 & n.d. & PG6 & 3 & + & - & - \\
\hline AG40 & $\mathrm{BH} 023810$ & PG6 & 5 & + & - & - \\
\hline PLG59 & n.d. & PG6 & 5 & + & - & - \\
\hline FG215 & BH023827 & PG6 & 8.7 & FG3 & 36.5 & 1 \\
\hline AC50 & BI203094 & PG6 & 17.5 & FG3 & 48.2 & 2 \\
\hline AC8 & BI203052 & PG6 & 34.5 & FGI & 61.2 & 2 \\
\hline PC2I & n.d. & PG6 & 56.4 & FGI & 72.8 & 2 \\
\hline PC73 & n.d. & PG6 & 64 & $\mathrm{~m}$ & - & - \\
\hline PC60 & n.d. & PG6 & 70 & + & - & - \\
\hline PgII & $X 75020$ & PG6 & 74.3 & FG6 & 22.8 & 1 \\
\hline LTP2 & n.d. & PG6 & 78 & + & - & - \\
\hline AC44 & BI203095 & PG7 & 10.3 & $\mathrm{FG} 2$ & 77.1 & 2 \\
\hline $\mathrm{PCI} 2$ & n.d. & PG7 & 24.7 & FG6 & 102.7 & 1 \\
\hline MC225 & BI203059 & PG7 & 28.4 & FG6 & 61.5 & 1 \\
\hline AGI04 & $\mathrm{BH} 023923$ & PG7 & 31.2 & FG6 & 57 & i \\
\hline TSA3 & n.d. & PG7 & 52.9 & FGI & 39.3 & 1 \\
\hline FG42 & n.d. & PG7 & 59 & $\mathrm{~m}$ & - & - \\
\hline FG27 & $\mathrm{BH} 023875$ & PG7 & 63 & + & - & - \\
\hline $\mathrm{CCl} 32$ & $\mathrm{BI} 203 \mathrm{I} 20$ & PG7 & 67.6 & FG3 & 38.3 & 2 \\
\hline FG24 & $\mathrm{BH} 023858$ & PG7 & 80 & ++ & - & - \\
\hline EXTI & n.d. & PG8 & 4 & ++ & - & - \\
\hline LY29 & $\mathrm{BH} 023873$ & PG8 & 20.8 & FG2 & 19.5 & 2 \\
\hline PCIOI & B1203099 & PG8 & 29.3 & $\mathrm{FG} 2$ & 19.7 & I \\
\hline FG37 & $\mathrm{BH} 02389 \mathrm{I}$ & PG8 & 40.9 & $\mathrm{FG} 2$ & 25.3 & i \\
\hline AG49 & $\mathrm{BH} 023870$ & PG8 & 49.1 & FG6 & 61.5 & 1 \\
\hline AC26 & $\mathrm{BI} 203074$ & PG8 & 52 & ++ & - & - \\
\hline Prul & X78119 & PG8 & 53.1 & $\mathrm{FG} 2$ & 26.7 & I \\
\hline PC36 & n.d. & PG8 & 60 & + & - & - \\
\hline
\end{tabular}

aLG $=$ Linkage group in Fragaria $(F)$ and Prunus $(P)$

n.d. $=$ not determined

$c+=$ no or weak hybridization

$\mathrm{e}_{\mathrm{m}}=$ monomorphic detected, and the total genetic distance covered by the $\mathrm{FV} \times \mathrm{FN}$ map was of $568.8 \mathrm{cM}$ with an average density of $2.5 \mathrm{cM} /$ marker and a ratio of $0.30 \mathrm{Mb} / \mathrm{cM}$. The resulting map shows minor rearrangements in comparison to the original obtained by Sargent et al. [18] most of which occurred in the upper part of the FG2. This map is presented in Additional file 1: 'FV $\times \mathrm{FN}$ reference map' that is included as supplementary information. The Prunus map included a total of 564 markers, the 562 described in [14] plus two SSRs mapped here. The map covers a distance of $511,3 \mathrm{cM}$ with an average density of $0.91 \mathrm{cM} /$ marker and a ratio of $0.59 \mathrm{Mb} / \mathrm{cM}$.

The distribution of the 71 anchor markers across the seven linkage groups of the strawberry genome was relatively even (Figure 1), ranging from seven markers on FG1 and FG5 to sixteen markers on FG2, with average marker densities ranging from $10.6 \mathrm{cM} /$ marker on FG1 to 5.5 on FG2. The map distance covered by these markers was $441.3 \mathrm{cM}$, a $78 \%$ of the map constructed with all markers. Only three large gaps of $>30 \mathrm{cM}$ were observed, one at the end of FG3 $(32.0 \mathrm{cM})$, one in the middle of FG2 $(31.7 \mathrm{cM})$ and the longest on the lower part of FG7 $(37.2 \mathrm{cM})$. The distribution of these 71 markers across the Prunus map was similar, with a maximum of 17 markers on PG1 and a minimum of four on PG4 and marker densities per linkage group ranging from 15.6 (PG4) to 5.0 (PG2) cM/ marker. Map coverage with anchor markers was of 399.5 $\mathrm{cM}$, a $78 \%$ of the complete map. Longest gaps between markers were smaller in Prunus, with only four exceeding 20 cM: 21.7 on PG7; 21.9 on PG6, 20.8 on PG8 and 28.6 on PG5. All the gaps on both the Fragaria and Prunus maps had at least one marker bin-mapped between the extreme markers of the gap, except for those on PG6 and FG3, suggesting that these were the longest gaps of each map.

\section{Map comparison between Prunus and Fragaria}

The overall pattern of synteny is summarized in Figure 2, where it can be seen that the majority of linkage groups of either species have most markers in one or two linkage groups of the other. The simplest case is G5 of both species which have all markers in common, and the most complex is FG6 which contains markers from five different Prunus linkage groups, although most of the markers on this linkage group belong to PG3 and PG7. Considering only the Prunus linkage groups, five of them contain most or all markers from only one group of Fragaria (PG2FG7, PG3-FG6, PG4-FG3, PG5-FG5 and PG8-FG2), and the other three of two groups (PG1-FG2 and FG4, PG6FG1 and FG3, and PG7-FG1 and FG6).

The presence of markers from two or more linkage groups of one species in a linkage group of the other would suggest that a fission/fusion, or a translocation event has 
Table 2: Gene and EST-based markers mapped in Prunus and Fragaria.

\begin{tabular}{|c|c|c|c|c|c|c|c|c|c|}
\hline \multirow[b]{2}{*}{ Locus } & \multicolumn{3}{|c|}{ Prunus } & \multicolumn{3}{|c|}{ Fragaria } & \multicolumn{3}{|c|}{ TBLASTX } \\
\hline & $\begin{array}{l}\text { Accession } \\
\text { number }\end{array}$ & $L^{\prime a}$ & $c M^{b}$ & $\begin{array}{l}\text { Accession } \\
\text { number }\end{array}$ & $\mathrm{LG}^{\mathrm{a}}$ & $c M^{b}$ & E-value ${ }^{c}$ & Swissprot-homologyd & Markere \\
\hline EFvVB2II9 & BU048565 & PGI & 4.3 & CX662119 & FG6 & $22.5-43.2$ & $5,00 \mathrm{E}-49$ & small GTP binding protein & SNP \\
\hline EFvVB2179 & BU0464I 4 & PGI & 4.3 & CX662179 & FG4 & $0.0-26.0$ & $2,00 \mathrm{E}-78$ & Aldoketo-reductase & SNP \\
\hline EFvNH8894 & BU042720 & PGI & 40.5 & DV438894 & FG2 & $53.9-73.8$ & $3.00 \mathrm{E}-84$ & auxin-induced protein (Aux22) & SNP \\
\hline EFaUF6868 & BU0468I7 & PGI & 48.0 & CO8I6868 & FG2 & $53.9-73.8$ & $1.00 \mathrm{E}-109$ & $20 S$ proteasome alpha 6 subunit & Indel \\
\hline EFaTRI976 & BU03976I & PGI & 48.6 & $\mathrm{CO} 381976$ & FG2 & $53.9-73.8$ & $2.00 \mathrm{E}-88$ & Mannan endo-I.4-Beta-Mannosidase & SNP \\
\hline EKO & AF495728 & PGI & $35.7-49.8$ & AY462247 & FG2 & 28.9 & 8.00E-I55 & Ent-kaurene oxidase & Indel \\
\hline DFR & AB095030 & PGI & $35.7-49.8$ & AY575057 & FG2 & 70.5 & $2.00 \mathrm{E}-106$ & dihydroflavonol reductase & Indel \\
\hline EFvVBI23I & BU046687 & PGI & 41.3 & CX66123I & FG4 & $26.0-46.1$ & $5.00 \mathrm{E}-17$ & RAD23-like & SNP \\
\hline EPpCU7308 & BU047308 & PGI & 75.2 & - & FG2 & $26.7-45.8$ & $5.00 \mathrm{E}-27$ & electron carrier/iron ion binding & Indel \\
\hline EPpCU9642 & BU039642 & PGI & 40.5 & - & FG2 & $0.0-26.7$ & 4.00E-72 & $\mathrm{ACT}$ domain-containing protein & Indel \\
\hline EFaUF7699 & BU040484 & PG2 & 7.9 & CO8I7699 & FG4 & $0.0-26.0$ & I.00E-102 & Luminal Binding Protein BiP & Indel \\
\hline EFaUF7084 & BU046792 & PG2 & 24.3 & $\mathrm{CO} 817084$ & FG7 & $63.4-81.0$ & $3.00 \mathrm{E}-96$ & $40 \mathrm{~S}$ ribosomal protein & SNP \\
\hline EPpCU2875 & BU042875 & PG2 & 25.0 & - & FG7 & 20.3 & $4.00 \mathrm{E}-132$ & RNA helicase & Indel \\
\hline EFvNH8484 & BU041902 & PG2 & 39.4 & DV438484 & FG7 & $27.0-38.6$ & $5.00 \mathrm{E}-105$ & GTP-Binding protein & SNP \\
\hline EPpCU9223 & BU039223 & PG2 & 39.4 & DY672045 & FG7 & 44.5 & I.00E-48 & 6-phosphofructokinase & Indel \\
\hline $\mathrm{ACO}$ & AFI 29073 & PG3 & 35.0 & AY706156 & FG6 & 83.1 & I.00E-165 & ACC oxydase & SSR \\
\hline EFvVB20I3 & BU039972 & PG5 & 0.0 & CX662013 & FG5 & $50.4-72.5$ & $6.00 \mathrm{E}-83$ & Pectinacetylesterase precursor & Indel \\
\hline ANS & AB097216 & PG5 & $|5.2-2| .0$ & AY695818 & FG5 & 9.7 & $4.00 \mathrm{E}-143$ & Anthocyanidin shynthase & Indel \\
\hline CEL-2 & AJ890498 & PG5 & $21.7-40.7$ & AF0546I5 & FG5 & 29.2 & 0.0 & endo-beta-I,4-glucanase & Indel \\
\hline AMPAII2 & AY377916 & PG5 & 4.1 & - & FG5 & 64.5 & - & -- & SSR \\
\hline EFvNH9852 & BU040757 & PG6 & 6.4 & DV439852 & FG7 & $27.0-38.6$ & I.00E-137 & 60S Ribosomal Protein LI0 & SNP \\
\hline EPpCU9257 & BU039257 & PG6 & 17.5 & - & FG7 & 24.9 & $7.00 \mathrm{E}-109$ & phosphoglucomutase precursor & Indel \\
\hline EPpCUI785 & BU04I785 & PG6 & 79.6 & DY669394 & FGI & $45.2-47.9$ & $1.00 \mathrm{E}-12$ & SNF4 (Sucrose NonFermenting 4) & SNP \\
\hline EPpCUI830 & BU04I830 & PG6 & 79.6 & CX661290 & FG6 & 14.1 & I.00E-132 & $26 \mathrm{~s}$ proteasome aaa-atpase subunit $\mathrm{rpt} 5 \mathrm{a}$ & Indel \\
\hline APX & EE488I29 & PG6 & $4.1-24.9$ & AFI 58654 & FG3 & 49.5 & 4.00E-102 & L-ascorbate peroxidase & Indel \\
\hline EFaUF7248 & BU043308 & PG7 & 10.3 & CO8I7248 & FG2 & $53.9-73.8$ & I.00E-97 & Methionine synthase & Indel \\
\hline EFvVBI923 & BU039764 & PG7 & 29.6 & CX661923 & FG6 & $56.5-68.1$ & $2.00 \mathrm{E}-73$ & Enolase & SNP \\
\hline EPpCU9910 & BU039910 & PG7 & 64.7 & - & FGI & 8.3 & $1.00 \mathrm{E}-62$ & putative ethanolamine kinase I & Indel \\
\hline $\mathrm{F} 3 \mathrm{H}$ & AB097I5I & PG7 & $42.5-47.8$ & $A B 201760$ & FGI & 40.6 & $2.00 \mathrm{E}-178$ & flavanone 3-hydroxylase & Indel \\
\hline PES & X95991 & PG7 & $49-56.1$ & AY324809 & FGI & 33.7 & 0.0 & Pectinesterase & Indel \\
\hline $\mathrm{ADH}$ & BU573880 & PG8 & $0.0-10.9$ & $X \mid 5588$ & FG2 & 17.9 & 4.00E-10I & alcohol dehydrogenase & SNP \\
\hline
\end{tabular}

Gene or EST-based and SSR markers mapped in the Prunus and Fragaria genomes, with their accession numbers, map positions and homology with known proteins of other species.

a $L G=$ linkage group

b Map position: If the marker was bin-mapped, the interval covered by the bin where the marker is located

c E-value at NCBI database restricted to plant sequences

d Predicted function

e Marker type detected

taken place between the two species since their divergence from a common ancestor. Nine of these rearrangements would have taken place between the Fragaria and the Prunus genomes, based on our results.

Colinearity of markers within syntenic regions of Prunus and Fragaria was only partial. The two most colinear groups FG5 and PG5 require only one inversion event to place all markers in the same order, but many more are required in other linkage groups. As shown in the materials and methods section, FG1 would require two inversions for the markers present on that linkage group to be in the same order as on PG7 and PG6 of Prunus, FG3 and FG4, three inversions, FG7 four inversions, FG6 six inver- sions and FG2 eight inversions. In total, at least 27 inversions are needed to account for the differences in the marker order of the two genomes. The estimated lower boundary of the total number of breakpoints that separate the Fragaria and Prunus genomes is thus 36 (nine translocations and 27 inversions).

A scheme of the possible evolution of the Fragaria and Prunus genomes from a hypothetical ancestral genome of $x=9[23]$ is presented in Figure 3 . In this scheme we have only considered the major chromosomal rearrangements, i.e. fusions/fissions and reciprocal translocations involving more than two of the markers used for the comparison. According to this scenario, the ancestral genome 
Table 3: Anchor markers used for map comparison.

\begin{tabular}{|c|c|c|c|}
\hline Markers & Prunus $(\mathrm{T} \times \mathrm{E})$ & Fragaria $(\mathrm{FV} \times \mathrm{FN})$ & No. anchor markers \\
\hline RFLPs & Mapped by Dirlewanger et al. (2004) & Mappeda & 40 \\
\hline Fragaria ESTs & Mapped in the transcript map (GDR) & Bin mappeda & 13 \\
\hline Fragaria ESTs & Bin mappeda & Mapped by Sargent et al. (2007) & 8 \\
\hline Prunus ESTs & Mapped in the transcript map (GDR) & Bin mapped $(3)^{\mathrm{a}}$ or mapped $(5)^{\mathrm{a}}$ & 8 \\
\hline Microsatellites & Mappeda & Mapped (I) by Sargent et al. (2007) or here (I) & 2 \\
\hline
\end{tabular}

Origin of the $7 \mathrm{I}$ anchor markers used for the comparison between the genomes of Prunus and diploid Fragaria using the reference populations ( $\mathrm{T} \times \mathrm{E}$ for Prunus and FV×FN for Fragaria) and mapping strategies used: with the whole population ("mapped") or by bin mapping ("bin mapped").

aResults obtained in this paper

underwent two fusions (A5/A6 and A7/A8, corresponding to FG6 and FG1, respectively) and a reciprocal translocation between part of the A8 chromosome in FG1 and A9, which resulted in FG3, to become the $x=7$ strawberry genome. For the Prunus $x=8$ genome, the ancestral genome was submitted to three fusions, A1/A2 to form PG1, A6/A7 to form PG7 and A8/A9 to form PG6 and two fissions, part of $\mathrm{A} 1$ in the $\mathrm{A} 1 / \mathrm{A} 2$ chromosome to form PG8 and part of A9 in the A8/A9 chromosome to form PG4.

Some inferences about the evolution of certain chromosomes can be formulated based on the comparison between the two maps (Figure 1), the proposed evolution of both genomes from an ancestral one (Figure 3), and the limited information available on cytogenetics and map comparison of the Rosaceae. The simplest comparison is that of FG5 and PG5, which display remarkable levels of structural conservation, the marker order between Prunus and Fragaria differing by just a single inversion event. Most markers of FG7 and PG2 are in common, suggesting their origin from a single ancestral chromosome (A4), but FG7 includes a short fragment of PG6, and PG2 is not completely included in FG7, with a small region being located on FG4. PG1 is a long linkage group that contains many more markers than the rest and that is likely to coincide with chromosome 1 of Prunus [14], which is clearly longer than the other chromosomes of this genus [24]. Based on karyotype observations, such a long chromosome does not exist in Malus [25] or in Fragaria [26]. The map comparison between Malus and Prunus [14] provided additional evidence that the long Prunus chromosome may be split into two in at least one of the constituent genomes of the amphidiploid Malus chromosome complement. Our data suggest that PG1 arose from the fusion of two ancestral chromosomes, A1 and A2, which correspond to FG4 and FG2, respectively, in strawberry. The region of PG1 where the fission/fusion occurred in the Prunus-apple comparison (38-44 cM from the top of PG1) is also compatible with that of the Prunus-Fragaria (estimated to be in the region of 25-45 cM from the top of PG1). Interestingly, most of PG8, one of the Prunus chromosomes with the fewest number of markers and with the smallest genetic distance, appears to be integrated into FG2, with five of the six anchor markers studied being in the same order in PG8 and FG2. This situation may be the result of the fission of A1 in Prunus but not in Fragaria.

\section{Discussion}

The comparison between the maps of Prunus and diploid Fragaria has been performed with 71 common markers. Most of them were mapped already in the Prunus map and were added to the strawberry map here. These markers resulted in good coverage of both genomes: $78 \%$ of the total distance of the reference maps of both Prunus [14] and strawberry [18]. The average density of anchor markers was of $7.3 \mathrm{cM} /$ marker with a maximum gap of $22 \mathrm{cM}$ (in PG6) for Prunus and $8.0 \mathrm{cM} /$ marker and a maximum gap of $32 \mathrm{cM}$ (in FG3) for Fragaria. The total map distance of the Prunus (519 cM) and the Fragaria (569 cM) maps were similar when constructed with all markers available $[14,18]$. Given that the genome size of Prunus is approximately twice that of strawberry these results suggest that the overall recombination rate per physical unit distance for the Fragaria $\mathrm{FV} \times \mathrm{FN}$ hybrid was higher than for the almond $\times$ peach $F_{1}$ individual that generated the $T \times E F_{2}$ population.

For the comparison between the two genomes, we selected 65 RFLP probes, all from Prunus or Malus species, which produced good hybridization and were single copy in the Prunus genome. These probes were studied in strawberry with the same stringency conditions as in Prunus and $16(24 \%)$ produced poor or no hybridization, suggesting that they were not present in the strawberry genome or that their sequences differed substantially from those of Prunus. Some of the probes that did not hybridize were located together in the same regions of the Prunus genome where several probes covering short genetic distances $(<10 \mathrm{cM}$ per region) did not hybridize, such as the central region of PG1 and the upper extreme of PG6 (Table 1). The three probes that did not hybridize in the PG1 region were two from a peach cDNA library (PC85 and PC15) and one from a genomic library of $P$. ferganensis (FG16). Two of them (PC15 and FG16) were 


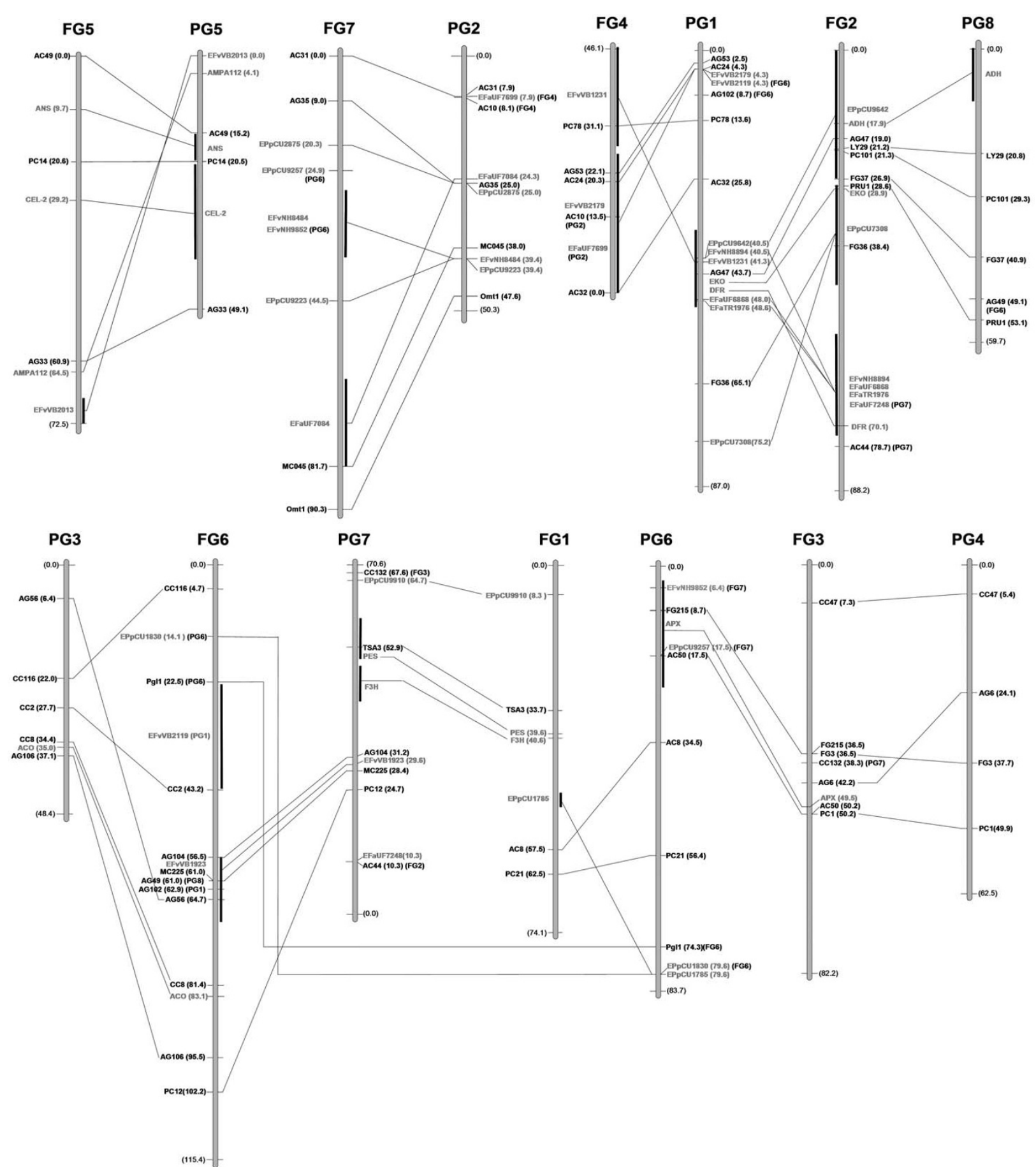

Figure I

Prunus-Fragaria map comparisons. Comparison between the maps of diploid Fragaria (FGI to FG7) and Prunus (PGI to PG8). Only common markers have been included in the framework of the reference maps of both genera. In parentheses after the markers is the distance from the origin of the linkage group to the marker for markers that have been mapped using the whole Prunus or Fragaria population. RFLP names are written in black and EST-derived markers in grey. The distance from the origin is not shown for markers that were bin-mapped, which are located within the region of the bin, indicated by a solid vertical bar at the corresponding locations on each of the linkage groups. Markers of one linkage group with correspondence in the other genome to linkage groups other than those that are in the neighbourhood have been indicated by the name of the corresponding group in parenthesis. 


\section{Prunus}

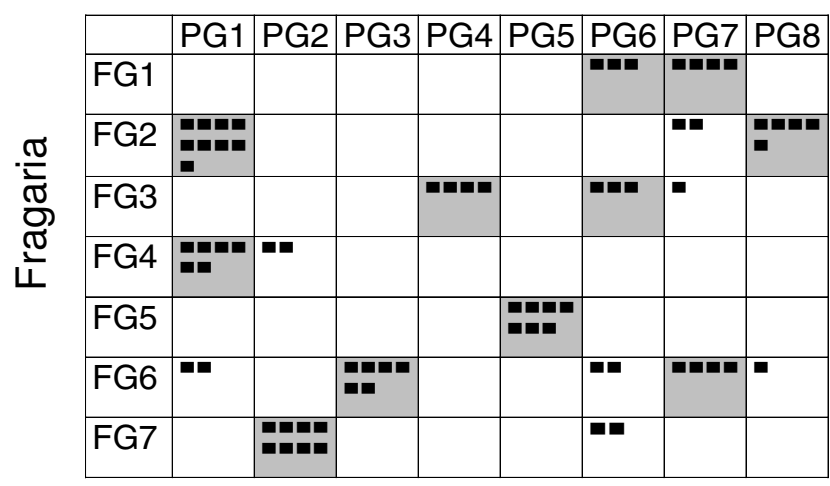

Figure 2

Prunus-Fragaria synteny comparison. Synteny between Prunus and Fragaria. Number of markers of each linkage group of one genus that correspond to the linkage groups in the other genus. Each marker is indicated by a black dot. Cells that contain three or more markers are noted with a grey background.

sequenced (Table 1) and had homology with known proteins. The region of PG6 had four markers (AG13, AG54, AG40 and PLG59), all of them from Prunus genomic libraries. Only AG40 was sequenced and its sequence had no homology with protein sequences. These results suggest that the corresponding regions, or at least the specific sequences tested, may be deleted in the strawberry genome. Given that some of the probes are homologous to proteins that are usually present as gene families such as polygalacturonase (FG16) and defensin protein 1 (PC15), an alternative explanation is that they may correspond to copies of these genes with high sequence divergence from those present in strawberry.

From the 40 RFLP probes that produced good hybridization and that were polymorphic, 14 (35\%) detected two loci in strawberry, whilst in Prunus they were single-locus (Table 1). Tanksley et al. [27] found that five (12\%) of the 42 tomato cDNA probes which they mapped in pepper had a different number of copies in each species. These differences in copy number may be due to differential deletions of duplicated DNA fragments existing in the ancestral genome from which Prunus and Fragaria originate. The presence of these duplications complicates the genome comparison because, if only one locus of the two can be mapped, as with the RFLPs mapped here in Fragaria, in half of the cases, the position of the marker could be interpreted as the presence of a spurious genetic rearrangement.
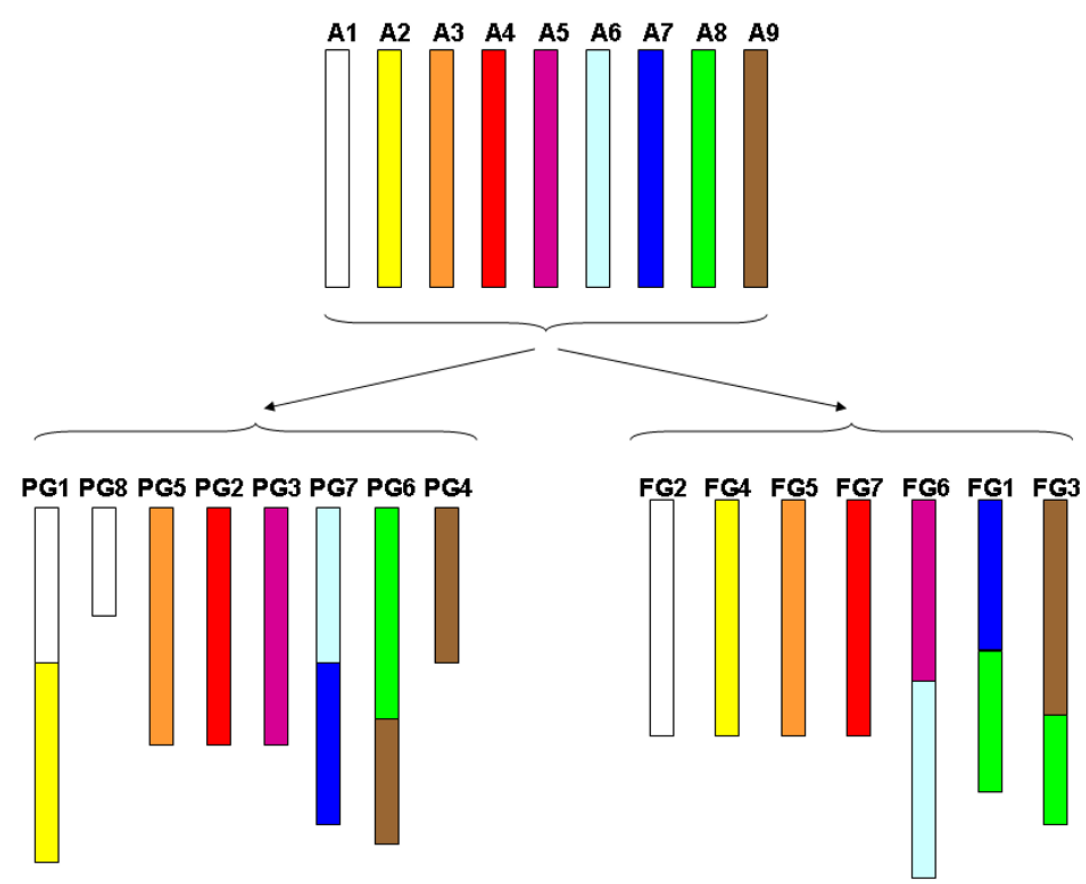

Figure 3

Scheme of the evolution of Prunus and Fragaria chromosomes from an ancestral genome. Model of evolution of Prunus (PGI-PG8) and Fragaria (FGI-FG7) chromosomes from a hypothetical ancestral Rosaceae genome with $x=9$ chromosomes (AI-A9). Only major chromosomal rearrangements (fusion/fission or translocation events involving more than two common markers between the two genomes) have been considered. 
The number of RFLPs mapped in $\mathrm{T} \times \mathrm{E}$ that were single copy, detected with Rosaceae probes, segregated in the strawberry population and had a good distribution along the Prunus map, was insufficient for a good coverage of the Prunus genome. This was due in part to the fact that approximately half of the probes used for RFLP mapping in Prunus have more than one copy $[28,29]$ and that a substantial number of probes used in $\mathrm{T} \times \mathrm{E}(30 \%)$ come from families other than the Rosaceae [14]. To solve this problem, we used the available EST and physical map information in Prunus to find ESTs placed in most of the uncovered regions. We then mapped these ESTs by an efficient and expensive approach, resequencing, using a cheap mapping strategy, bin mapping. This allowed us to cover most gaps of the Prunus genome and to reduce the maximum gap without anchor markers in this species to $22 \mathrm{cM}$. Given the fast rate of growth of the information on the Prunus transcript map, this strategy is likely to allow us a more detailed analysis of synteny in the near future.

Figure 2 shows that the level of synteny between Prunus and Fragaria is high, with most of the markers that mapped to the same linkage group in one species mapping to one or two linkage groups in the other. However, the colinearity is only partial, with an estimated number of nine chromosomal rearrangements involving two chromosomes (translocations or fusion/fission events) and 27 inversions. The number of translocations and fusion/fission events is high if we compare it with other confamilial comparisons where this number has been estimated. Only the distant Solanaceae genomes of pepper and tomato, with 10 of these rearrangements estimated [30], yielded similar results. However, the predominance of inversions over other rearrangements that we found in the PrunusFragaria genome comparison is frequent in the Plant Kingdom, such as in the comparisons between tomato and potato [31], tomato and eggplant [2], tomato and pepper [30], Brassica nigra and Arabidopsis thaliana [32] or a. thaliana and A. lyrata [33].

The centromeres or heterochromatic regions around the centromeres are often where breakpoints occur, leading to chromosomal rearrangements. Most inversions and translocations in the Solanaceae [30,34] and Poaceae [35] had their breakpoints at or near the centromere. This information may be useful to deduce the position of the centromeres of some of the Prunus or Fragaria chromosomes. For example, based on the position where we hypothesize that a fusion event occurred between ancestral chromosomes A1 and A2 to form PG1, we may infer that the centromere is located in the central part of this chromosome (30-45 cM from the top). This is consistent with the metacentric nature of peach chromosome 1. Schubert [36] proposed a model for chromosome fusion where a reciprocal translocation between an acrocentric or telocentric chromosome and another chromosome may generate a larger fused chromosome plus a small chromosome that is eventually lost. Following this model, one of FG2, FG4 or both, or their ancestral chromosomes (A1 and A2), was probably acrocentric. Other metacentric chromosomes may be those that are composed of parts (possibly entire translocated arms) of two chromosomes of the other species; this may be the case of PG7, PG6, FG1, FG6 and FG3 (see Figure 3). In the case of PG6, a reciprocal translocation with PG8 was previously reported in peach [37]. The breakpoint was estimated to be located in the region of 18-39 cM from the top of PG6, which coincides with the region of junction between the fragments of FG1 and FG3 $(18-35 \mathrm{cM})$ and may correspond to centromeric regions of these three chromosomes. On the other hand, the translocation breakpoint of PG8 [37] is located in its distal region, suggesting that it is an acrocentric chromosome.

A rate of $0.14( \pm 0.06)$ structural mutations per chromosome per million years (My) of divergence was estimated [6] from the analysis of various macrosynteny comparisons in plants. Considering this rate, our estimation of a number of at least 36 chromosomal rearrangements between Prunus and Fragaria, assuming that the initial number of chromosomes of the common ancestor was $x$ $=9$, allows us to estimate that the divergence of these two species dates from approximately 29 Mya. This places these two genera as distant taxa within the Rosaceae, similar to maize and sorghum in the Poaceae ( 24 Mya) but closer than tomato and pepper in the Solanaceae ( $\sim 40$ Mya), maize and rice or wheat and rice, both with an estimated divergence time of $\sim 66$ Mya [6].

Our results indicate that there is sufficient synteny between the genomes of Fragaria and Prunus to allow the information on marker or gene or quantitative trait locus (QTL) position from one of these species to be used in the other. For example, the gene that determines the ability to produce runners (vegetative propagules) in strawberry $(R /$ $r$ ) is located on FG2 at a position syntenic to the region of PG1 where the Evergrowing gene (Evg/evg) that determines continuous leaf production [38] and a QTL that determines blooming time in peach are located [39]. The gene for seasonal vs. perpetual flowering in strawberry $(S /$ s) maps to a region of FG6 (proximal to the SSR EMFn017), that in our comparison roughly coincides with a PG7 fragment where a major QTL determining blooming time in peach lies [39]. These comparisons are however preliminary and need to be studied in more detail, but are a first insight into other possible comparisons that may facilitate the advancement on the knowledge of the genetics of key characters of the Rosaceae. 


\section{Conclusion}

Whilst the economical importance of peach and its relatively easy manipulation (shorter intergeneration period and self-compatibility) compared to other fruit tree species have determined that many genes have been studied [40] and that the position of at least 28 of them has been established on the Prunus map [14], the diploid strawberry has important advantages, i.e., a genome of a size similar to that of Arabidopsis, ease of genetic transformation, and a rapid life-cycle. In addition, the plants are small, and produce a large number of seed per cross, and thus diploid strawberry may become a very efficient organism for reverse genetics and other genomics applications that may provide useful information for other Rosaceous species, particularly fruit tree crops [41]. This will be facilitated by the information on map comparisons between these two genera that we present in this paper.

\section{Methods \\ Plant material}

The parents and progeny of two mapping populations were used for comparing the Prunus and Fragaria genomes, those of the $\mathrm{F}_{2}$ interspecific Prunus reference mapping population $(\mathrm{N}=82)$ derived from the $\mathrm{F}_{1}$ cross between the almond ( $P$. dulcis) cultivar 'Texas' and the peach $(P$. persica) cultivar 'Earlygold' (abbreviated T×E) and the $\mathrm{F}_{2}$ interspecific diploid Fragaria reference mapping population $(\mathrm{N}=76)$ derived from the $\mathrm{F}_{1}$ cross of $F$. vesca $815 \times$ F. nubicola 601 (abbreviated $\mathrm{FV} \times \mathrm{FN}$ ). The $\mathrm{T} \times \mathrm{E}$ map is composed of 562 markers (185 SSRs, 361 RFLPs, 11 isoenzymes and 5 STSs) [14], whilst the $\mathrm{FV} \times \mathrm{FN}$ map currently consists of 182 markers (175 SSRs, 6 gene specific markers and 1 SCAR) [18]. Most markers used were selected from $\mathrm{T} \times \mathrm{E}$ and subsequently mapped in $\mathrm{FV} \times \mathrm{FN}$.

\section{DNA extraction}

For Fragaria, one gram of young expanding leaves of each individual was collected and kept at $-80^{\circ} \mathrm{C}$ before DNA isolation. Genomic DNA was isolated from the leaf samples using the CTAB method of Doyle and Doyle [42], followed by DNeasy miniprep kit purification (Qiagen). DNA concentrations were measured using a Gene Quant II spectrophotometer (Pharmacia Biotech). For Prunus, DNA was extracted as described in [28].

\section{RFLP markers}

A total of 65 probes from various Prunus species and apple were used for RFLP analysis (Table 1). These probes were selected from those used in the construction of the Prunus reference map [14] to be single-copy and covering the whole genome at approximately even distances of 10-25 cM. RFLP analysis was performed with the procedure of Viruel et al. [28]. DNA probes were first hybridized in the parents of the $\mathrm{FV} \times \mathrm{FN}$ population and those that detected RFLPs were studied later in all $\mathrm{F}_{2}$ individuals.

\section{Markers based on Fragaria or Prunus ESTs}

An additional set of markers was developed from the Prunus transcript map, i.e. the collection of EST unigenes that are located on the same BAC or the same BAC contig of the peach physical map as mapped markers (usually RFLPs) of the reference linkage map [43]. We analysed 515 T×E ESTs currently anchored in the Prunus transcript map (found in the Genome Database for Rosaceae) to find homologous EST sequences in Fragaria by using the TBLASTX program [44]. The selected sequences (only sequences with an e-value $<1.00 \mathrm{E}-15$ ) (Table 2) were then analyzed with TBLASTN, and the most homologous regions were selected for primer design. Primers were designed to include putative Fragaria introns based on the Arabidopsis genome sequence [45] and to give an expected amplicon size $>800 \mathrm{bp}$, using the program Primer3 [46].

For SNP detection, Fragaria ESTs were PCR-amplified in the parents of the Fragaria mapping population in a temperature-gradient PCR to obtain the optimum annealing temperature for each EST primer pair. The PCR reactions were performed as described in [47] and amplification products were sequenced with an ABI PRISM 3700 DNA Analyzer and SNPs were detected between the two parental lines through sequence alignment using the STADEN package [48] and verified by visual inspection of the DNA chromatograms. The same procedure was followed for Prunus ESTs of the transcript map for which we did not find homologous Fragaria ESTs.

The sequences of the primers used for the development of these markers can be found in Table 4. The terminology used for the EST derived markers was: E (for EST), a two letter code for the species where the EST was obtained (Fv, Fa or Pp for F. vesca, F. $x$ ananassa and P. persica, respectively), two additional letters for the place where the EST was obtained (i.e. NH for University of New Hampshire, VB for Virginia Bioinformatics Institute, UF for University of Florida, TR for Trisaia Research Center and CU for Clemson University) and four numbers that correspond to the last four digits of the EST EMBL accession number.

\section{Fragaria gene-specific markers}

Eight further gene specific loci that had previously been mapped in the $\mathrm{FV} \times \mathrm{FN}$ population [21] were mapped in the $\mathrm{T} \times \mathrm{E}$ population. The primer pairs used for amplification of four of these genes (CEL-2, PES, ANS and APX) were previously described by Sargent et al. [21] whilst primer pairs for the remaining four (EKO, F3H, DFR and $\mathrm{ADH}$ ) were designed following the procedures of Sargent et al. [21] from gene sequences deposited in the EMBL database and are listed in Table 4 . The primer pairs were used to amplify products from the parents of the Prunus 
Table 4: Gene and EST-based marker primer sequences. Primers used for DNA amplification in the markers obtained from Fragaria gene or EST or Prunus EST sequences.

\begin{tabular}{|c|c|c|c|c|}
\hline Origin & Locus name & Forward primer sequence $5^{\prime}-3^{\prime}$ & Reverse primer sequence $5^{\prime}-3^{\prime}$ & Reference $^{a}$ \\
\hline \multirow[t]{4}{*}{ Fragaria gene } & EKO & ACAGTCCAGCTCCAATAGTTCC & GCTTTCCCATTGATTCTTGTCC & AY462247 \\
\hline & $\mathrm{F} 3 \mathrm{H}$ & GAGTTGATACCAAGCTCATCTCG & GTCAССTСTСTCСАTССTTCC & AB09715I \\
\hline & DFR & CCACTCCTATGGATTTTGAGTCC & CTAGCACCCCATTTATTGTTGG & $\mathrm{AB} 095030$ \\
\hline & $\mathrm{ADH}$ & GCKTCAMGAATTATYGGKGTTG & ATGGGASTTKRTGGGTGATG & $\underline{X 15588}$ \\
\hline \multirow[t]{15}{*}{ Fragaria EST } & EFvVB2179 & ATCTGCGTGACAATGCAAAG & AAGAGCCTTCAGTTGCTCCA & CX662179 \\
\hline & EFvVB21I9 & GCTCGAGCTGATTACGATTACC & TAAAGGACCCATCAGAGAAACG & CX662119 \\
\hline & EFvNH8894 & GGTTAGGTCTCCCCAGTGGT & GGACGTCTCCCACTAGCATC & DV438894 \\
\hline & EFvVBI23I & CСAACTGTGACATCCACGAC & GCTGTCACGCAGAAAATCAA & CX661231 \\
\hline & EFaUF6868 & GCTCTTCCAGGTCGAGTACG & GTTTCCACTTGGGCAGTTGT & $\overline{\mathrm{CO} 816868}$ \\
\hline & EFaTRI976 & GTTGGTGCTGAGTTTGGTGA & CCCAACTGCTCAAGAAGGAG & $\overline{\mathrm{CO} 381976}$ \\
\hline & EFaUF7699 & GTTCTTGTTGGTGGAAGCAC & ССTCAAAGACCTGAATGGAG & $\mathrm{CO} 817699$ \\
\hline & EFaUF7084 & CAGAAGAGGTTCAAGTTCC & ACACCATAGCAAGCCCTG & $\mathrm{CO} 817084$ \\
\hline & EFvNH8484 & TTCTGGTGTCGGCAAGTC & AGGCCTGCTCAACATTGG & DV438484 \\
\hline & EFvVB20I3 & GTGCAGTTGCCAAAGGAGC & AGCTGGGTTTGCTGCTT & $\overline{\mathrm{C} \times 662013}$ \\
\hline & EFvNH9852 & TTCTGTCGTGGTGTCCC & ATGATCTTTTGGCGACCA & $\overline{D V 439852}$ \\
\hline & EFvNH7822 & GATGCTGGGTCTGCTGGG & GCCTGCTCATTGGCATA & $\mathrm{CO} 817822$ \\
\hline & EFaUF7248 & ACTGCTCGCCCAATGAAG & TCACATCAGCATCCATGTCA & $\overline{\mathrm{CO}} \mathrm{BI7248}$ \\
\hline & EFavBI 923 & GGCCGTGTCTCTTGCAGT & TGGGAGCAAATCCACCTT & CX661923 \\
\hline & $\mathrm{ACO}$ & AGCACCTTCTACCTCAAACACC & CTCACAGAACAAGTCCAAGAGC & $\underline{\mathrm{AFI}} 29073$ \\
\hline \multirow[t]{8}{*}{ Prunus EST } & EPpCU9642 & TTCAGTTGGCAGATCCTGTG & TGCTGAGACCCTTCCAATTT & $\overline{\text { BU039642 }}$ \\
\hline & EPpCUI785 & TTTTCCAAACCTTGCTGGAG & GCAGTAGCTGTGGCAATGAA & $\overline{\text { BU04|785 }}$ \\
\hline & EPpCU7308 & GGCAGGCCGCTCTTATACTA & GACTCTTTTCGGGGTTCCA & BU047308 \\
\hline & EPpCU9257 & CACCACCGTTTCAAAAGAGG & CTGAAGCTCTAGCTGAGGCAAG & BU039257 \\
\hline & EPpCUI830 & TGATGCAATTGGCACAAAGC & ССТАТСАССАСТTАСТTСАСТGC & BU041830 \\
\hline & EPpCU9910 & ATAACTCTGCCATCCGAATCC & CATTCCTTGAACAGATCCTTGC & $\overline{B \cup 039910}$ \\
\hline & EPpCU9223 & AACAGAGCCAAGCTTATGCAG & TTTCTGCGCAACCGCATC & BU039223 \\
\hline & EPpCU2875 & AACTCAGAGACATATCTGCACAGG & AAGTTGAAGCGGTCTTCATAGG & BU042875 \\
\hline
\end{tabular}

${ }^{a} E M B L$ accession numbers for the sequences from which novel primer pairs were designed

mapping population following the procedure of Sargent et al. [47] and SNPs were detected as described above.

\section{Microsatellite markers}

One SSR (ACO) developed in the 5' UTR of Prunus 1-aminocyclopropane-1-carboxylate oxidase gene (ACC oxidase; AF129073) was used to locate this gene, which was already mapped in Fragaria [21], onto the T×E map. The primers used are listed in Table 4. Another apricot SSR, AMPA112, previously described by Hagen et al. [49], was polymorphic in both reference populations and was also included in the map comparison. PCR reactions were performed as described by [50].

\section{Map construction in Fragaria and bin mapping in Prunus and Fragaria}

The T×E map of Dirlewanger et al. [14], constructed with MapMaker/EXP v. 3.0 [51], was used as a standard for map comparisons. Data for the $\mathrm{FV} \times \mathrm{FN}$ map, originally constructed with Joinmap 3.0 [52] by Sargent et al. [18], was used for the mapping of novel loci in Fragaria. However, in order to make both maps comparable, the same data of the $\mathrm{FV} \times \mathrm{FN}$ map plus those obtained here were used, but the map was reconstructed with MapMaker. The
Kosambi mapping function was used to convert recombination units into genetic distances. The mapping procedure followed the guidelines of previous maps constructed in Prunus [29]. The usual notation for the eight linkage groups of Prunus is G1 to G8 and for the seven Fragaria groups is I-VII. In order to facilitate the map comparison we have used on this occasion the terminology PG1-PG8 for Prunus and FG1-FG7 for Fragaria.

A subset of six plants of the T×E mapping population having a high number of recombination breakpoints and a uniform distribution across the Prunus genome was selected by Howad et al. [15]. The genotype of this set of plants (the bin set) identified 64 fragments (bins) of the Prunus map with average size $7.8 \mathrm{cM}$. Following a similar approach, a bin set of six plants was selected from the FV $\times$ FN mapping population by Sargent et al. [22]. The Fragaria bin set detected 46 fragments of its genome with an average length of $12.6 \mathrm{cM}$. Using the bin sets of both species allowed us to establish the position of some of the markers with lower cost and effort than mapping with the whole population. 


\section{Mapping strategy}

Table 3 summarizes the mapping strategy employed for all novel markers mapped in this investigation. All RFLPs used were already mapped in Prunus using the entire $\mathrm{T} \times \mathrm{E}$ progeny and were mapped in Fragaria using the entire FV $\times$ FN population. The Fragaria and Prunus ESTs found to be polymorphic in the parents of the Fragaria mapping population were either sequenced in the Fragaria bin set and bin-mapped, or mapped in the whole $\mathrm{FV} \times \mathrm{FN}$ population when clear intron length polymorphisms were detected. The eight Fragaria ESTs, previously mapped in FV $\times$ FN, were bin mapped in Prunus. The SSRs were mapped in Prunus and Fragaria using all individuals of these populations. The data for each marker were scored independently by two researchers. Conflicting results were re-examined and in case of disagreement, the most conservative option was taken.

\section{Estimating the number of chromosomal rearrangements}

To estimate the number of chromosomal rearrangements that have occurred between Prunus and Fragaria since they diverged from a common ancestor, we elaborated a list of the markers of each of the Fragaria linkage groups with their correlative position on the Prunus linkage map. Taking FG1 as an example, this linkage group has seven anchor markers with order 7.2 (PG7, position 2), 7.3, 7.5, $7.4,6.10,6.6,6.7$. Then, we deduced the minimal number of mutations that would place the positions of these markers in the same order as in Prunus. In the example of FG1, we counted one translocation (between PG7 and PG6) and two inversions, one involving the 7.4-7.5 fragment and the other the 6.6-6.7 fragment, to give the final marker order: 7.2, 7.3, 7.4, 7.5, 6.10, 6.7, and 6.6. An additional mutation (probably caused by a translocation) had to occur to explain the gap between 6.10 and 6.7. This mutation was considered when analyzing the chromosome that received the translocated fragment. We counted one breakpoint per translocation and one per inversion. This is a lower boundary, as inversions may require one breakpoint if they involve the distal part of a chromosome, or two if they correspond to an internal fragment of a chromosome. Finally, we considered as translocations only those regions comprising two or more markers, as single-locus translocations are more likely to be spurious.

\section{Authors' contributions}

AM and PA conceived and designed the experiments. SV carried out the experiments with RFLPs in the Fragaria mapping population, DJS performed experimental work for mapping gene-specific markers in the Fragaria and Prunus bin mapping populations, and AM carried out the experimental work for mapping EST in the Fragaria population. PA coordinated the preparation of the manuscript to which all authors contributed, and read and approved the final manuscript.

\section{Additional material}

\section{Additional file 1}

FV $\times$ FN reference map. The diploid Fragaria reference map constructed using MapMaker from the data of Sargent et al. (2006) and the novel markers (boldface and red) added in this paper.

Click here for file

[http://www.biomedcentral.com/content/supplementary/14712229-8-67-S1.pdf]

\section{Acknowledgements}

This research was supported in part with funds of the Spanish Ministry of Education project INIA (RTA2007-00063-00-00). Rosaceous genomics at East Malling is sponsored by Defra.

\section{References}

I. Tanksley SD, Ganal MW, Prince JP, De Vicente MC, Bonierbale MW, et al: High density molecular linkage maps of the tomato and potato genomes. Genetics 1992, I32: I |4 I-I I 60.

2. Doganlar S, Frary A, Daunay MC, Lester RN, Tanksley SD: A comparative genetic linkage map of eggplant (Solanum melongena) and its implications for genome evolution in the Solanaceae. Genetics 2002, I61:1697-1711.

3. Devos KM, Gale MD: Genome relationships: the grass model in current research. Plant Cell 2000, I 2:637-646.

4. Choi HK, Mun JH, Kim DJ, Zhu HY, Baek JM, Mudge J, Roe B, Ellis N, Doyle J, Kiss GB, Young ND, Cook DR: Estimating genome conservation between crop and model legume species. Proc Natl Acad Sci USA 2004, I 0 I: I 5289-I5294.

5. Schranz ME, Song BH, Windsor AJ, Mitchell-Olds T: Comparative genomics in the Brassicaceae: a family-wide perspective. Curr Opin Plant Biol 2007, I 0: I68-I75.

6. Paterson AH, Lan TH, Reischmann KP, Chang C, Lin YR, et al: Toward a unfied genetic map of higher plants, transcending the monocot-dicot divergence. Nature Genet 1996, I 4:380-386.

7. Salse J, Piégu B, Cooke R, Delseny M: Synteny between Arabidopsis thaliana and rice at the genome level: a tool to identify conservation in the ongoing rice genome sequencing project. Nucleic Acids Res 2002, 30:23 I 6-2328.

8. Dominguez I, Graziano E, Gebhardt C, Barakat A, Berry S, Arús P, Delseny $M$, Barnes S: Plant genome archaeology: evidence for conserved ancestral chromosome segments in dicotyledonous plant species. Plant Biotech J 2003, I:91-99.

9. Rong J, Bowers JE, Schulze SR, Waghmare VN, Rogers CJ, et al: Comparative genomics of Gossypium and Arabidopsis: Unraveling the consequences of both ancient and recent polyploidy. Genome Res 2005, I 5: I 198-1210.

10. Judd WS, Campbell CS, Kellogg EA, Stevens PF: Plant systematics, a phylogenetic approach Sunderland M.A. Sinauer Associates; 1999.

II. Akiyama Y, Yamamoto Y, Ohmido N, Oshima M, Fukui K: Estimation of the nuclear DNA content of strawberries (Fragaria spp.) compared with Arabidopsis thaliana by using dual system flow cytometry. Cytologia 2001, 66:431-436.

12. Arumuganathan K, Earle ED: Nuclear DNA content of some important plant species. Plant Mol Biol Rep 1991, 9:208-218.

13. Arús P, Yamamoto T, Dirlewanger E, Abbott AG: Synteny in the Rosaceae. In Plant Breeding Reviews Edited by: Janick J. London, John Wiley and Sons Inc; 2005:175-21I.

14. Dirlewanger E, Graziano E, Joobeur T, Garriga-Caldere F, Cosson P, Howad W, Arus P: Comparative mapping and marker-assisted selection in Rosaceae fruit crops. Proc Nat Acad Sci USA 2004, | 0 |:989|-9896.

15. Howad W, Yamamoto T, Dirlewanger E, Testolin R, Cosson P, Cipriani G, Monforte AJ, Georgi L, Abbott AG, Arus P: Mapping with a few plants: using selective mapping for microsatellite saturation of the Prunus reference map. Genetics 2005, I 7 |: | 305-1309.

16. Yamamoto T, Kimura T, Saito T, Kotobuki K, Matsuta N, Liebhard R, Gessler C, Weg WE van de, Hayashi T: Genetic linkage maps of 
Japanese and European pears aligned to the apple consensus map. Acta Hort 2004, 663:5 I-56.

17. Pierantoni L, Cho KH, Shin IS, Chidoni R, Tartarini S, Dondini L, Kang SJ, Sansavini S: Characterisation and transferability of apple SSRs to two European pear $F_{1}$ populations. Theor Appl Genet 2004, 109:1519-1524.

18. Sargent DJ, Clarke J, Simpson DW, Tobutt KR, Arus P, Monfort A, Vilanova S, Denoyes-Rothan B, Rousseau M, Folta KM, Bassil NV, Battey $\mathrm{NH}$ : An enhanced microsatellite map of diploid Fragaria. Theor Appl Genet 2006, I I 2: I349- I 359.

19. Jung S, Jesudurai C, Staton M, Du ZD, Ficklin S, Cho IH, Abbott $A$, Tomkins J, Main D: GDR (Genome Database for Rosaceae): integrated web resources for Rosaceae genomics and genetics research. BMC Bioinformatics 2004, 5:130 [http://www.bio info.wsu.edu/gdr/].

20. Potter D, Eriksson T, Evans RC, Oh S, Smedmark JEE, Morgan DR, Kerr M, Robertson KR, Arsenault M, Dickinson TA, Campbell CS Phylogeny and classification of Rosaceae. Plant Syst Evol 2007, 266:5-43.

21. Sargent DJ, Rijs A, Nier S, Simpson DW, Tobutt KR: The development and mapping of functional markers in Fragaria and their transferability and potentialfor mapping in other genera. Theor Appl Genet 2007, I | 4:373-384.

22. Sargent DJ, Cipriani G, Vilanova S, Gil-Ariza D, Arús P, Simpson DW Tobutt KR, Monfort A: The development of a bin mapping population and the selective mapping of 103 markers in the diploid Fragaria reference mapping population FV×FN. Genome 2008, 5 I: I $20-127$

23. Morgan DR, Soltis DE, Robertson KR: Systematic and evolutionary implications of the RBC $L$ sequence variation in Rosaceae. Amer J Bot 1994, 81:890-903.

24. Salesses G, Mouras A: Tentative d'utilisation des protoplastes pour l'etude des chromosomes chez les Prunus. Ann Amelior Plantes 1977, 27:363-368.

25. Bouvier L, Lespinasse $Y$, Schuster M: Karyotype analysis of an haploid plant of apple (Malus domestica). Acta Horticulturae 2000, 538:321-324.

26. Iwatsubo $\mathrm{Y}$, Naruhashi $\mathrm{N}$ : Karyotypes of three species of Fragaria (Rosaceae). Cytologia 1989, 54:493-497.

27. Tanksley SD, Bernatzky R, Lapitan NL, Prince JP: Conservation of gene repertoire but not gene order in pepper and tomato. Proc Natl Acad Sci USA 1988, 85:6419-6423.

28. Viruel MA, Messeguer R, de Vicente MC, Garcia-Mas J, Puigdomenech $P$, et al: A linkage map with RFLP and isozyme markers for almond. Theor Appl Genet 1995, 91 :964-97I.

29. Joobeur T, Viruel MA, de Vicente MC, Jauregui B, Ballester J, Dettori MT, Verde I, Truco M], Messeguer R, Batlle I, Quarta R, Dirlewanger E, Arus P: Construction of a saturated linkage map for Prunus using an almond × peach F2 progeny. Theor Appl Genet 1998, 97:1034-1041.

30. Livingstone K, Lackney VK, Blauth JR, Wijk RV, Jahn MK: Genome mapping in Capsicum and the evolution of genome structure in the Solanaceae. Genetics 1999, I52:1 | 83-1202.

31. Bonierbale MW, Plaisted RL, Tanksley SD: RFLP maps based on a common set of clones reveal modes of chromosomal evolution in potato an tomato. Genetics 1988, 120:1095-1103.

32. Lagercrantz U: Comparative mapping between Arabidopsis thaliana and Brassica nigra Indicates that Brassica genomes have evolved through extensive genome replication accom panied by chromosome fusions and frequent rearrangements. Genetics 1998, 150:1217-1228.

33. Yogeeswaran K, Frary A, York TL, Amenta A, Lesser AH, Nasrallah JB, Tanksley SD, Nasrallah ME: Comparative genome analyses of Arabidopsis spp.: Inferring chromosomal rearrangement events in the evolutionary history of A. thaliana. Genome Res 2006, I5:505-5I5.

34. Paterson AH, Bowers JE, Burow MD, Draye X, Elsik CG, liang CX Katsar CS, Lan TH, Lin YR, Ming RG, Wright RJ: Comparative genomics of plant chromosomes. Plant Cell 2000, I 2:1523-1539.

35. Moore G, Roberts M, Aragon-Alcaide L, Foote T: Centromeric sites and cereal chromosome evolution. Chromosoma 1997, 105:321-323.

36. Schubert I: Chromosome evolution. Curr Opin Plant Biol 2007, 10:109-II5.
37. Jáuregui B, de Vicente MC, Messeguer R, Felipe A, Bonnet A, Salesses $G$, Arus P: A reciprocal translocation between 'Garfi' almond and 'Nemared' peach. Theor Appl Genet 200I, I02:II69-1176.

38. Bielenberg DG, Wang Y, Fan S, Reighard GL, Scorza R, Abbott AG: A deletion affecting several gene candidates in present in the evergrowing peach mutant. J Hered 2004, 95:436-444.

39. Silva C, Garcia-Mas J, Sanchez AM, Arús P, Oliveira MM: Looking into flowering time in almond (Prunus dulcis (Mill) D.A. Webb): the candidate gene approach. Theor Appl Genet 2005, I I 0:959-968.

40. Monet R, Guye A, Roy M, Dachary N: Peach Mendelian genetics: a short review and new results. Agronomie 1996, I6:32I-329.

41. Folta KM, Davis TM: Strawberry genes and genomics. Critical Rev Plant Sci 2006, 25:399-4I5.

42. Doyle IJ, Doyle JL: A rapid DNA isolation procedure for small quantities of fresh leaf tissue. Phytochem Bull 1987, 19: I I-15.

43. Horn R, Lecouls AC, Callahan A, Dandekar A, Garay L, McCord P, Howad W, Chan H, Verde I, Main D, Jung S, Georgi L, Forrest S, Mook J, Zhebentyayeva T, Yu YS, Kim HR, Jesudurai C, Sosinski B, Arus P, Baird V, Parfitt D, Reighard G, Scorza R, Tomkins J, Wing R, Abbott AG: Candidate gene database and transcript map for peach, a model species for fruit trees. Theor Appl Genet 2005, I I 0:1419-1428

44. Atschul SF, Madden TL, Schaffer AA, Zhang J, Zhang Z, Miller W, Lipman DJ: Gapped BLAST and PSI-BLAST: a new generation of protein database search programs. Nucleic Acids Res 1997 25:3389-3402 [http://www.ncbi.nlm.nih.gov/BLAST/Blast.cgi].

45. Arabidopsis genome sequence [http://arabidopsis.org]

46. Primer3 [http://frodo.wi.mit.edu/cgi-bin/primer3/ primer3 www.cgi]

47. Sargent DJ, Hadonou AM, Simpson DW: Development and characterisation of polymorphic microsatellite markers from Fragaria viridis, a wild diploid strawberry. Mol Ecol Notes 2003, 3:550-552.

48. Staden R: The Staden sequence analysis package. Mol Biotechnol 1996, 5:233-24I.

49. Hagen LS, Chaib J, Fady B, Decroocq V, Bouchet IP, Lambert P, Audergon JM: Genomic and cDNA microsatellites from apricot (Prunus armeniaca L.). Mol Ecol Notes 2004, 4:742-745.

50. Monfort A, Vilanova S, Davis TM, Arús P: A new set of polymorphic simple sequence repeat (SSR) markers from a wild strawberry (Fragaria vesca) are transferable to other diploid Fragaria species and to Fragaria x ananassa. Mol Ecol Notes 2006, 6:197-200.

51. Lander ES, Gree P, Abrahansom J, Barlow A, Daly MJ, et al: MAP. MAKER: an interactive computer package for constructing primary genetic linkage maps of experimental and natural populations. Genomics 1987, I:|74-|81.

52. Van Ooijen JW, Voorrips RE: Joinmap 3.0:software for the calculation of genetic linkage maps. Plant research International, Wageningen, The Nederlands; $200 \mathrm{I}$

Publish with BioMed Central and every scientist can read your work free of charge

"BioMed Central will be the most significant development for disseminating the results of biomedical research in our lifetime. "

Sir Paul Nurse, Cancer Research UK

Your research papers will be:

- available free of charge to the entire biomedical community

- peer reviewed and published immediately upon acceptance

- cited in PubMed and archived on PubMed Central

- yours - you keep the copyright

Submit your manuscript here:

http://www.biomedcentral.com/info/publishing_adv.asp
BioMedcentral 